THE EFFECT OF SURFACE TENSION IN MODELING INTERFACIAL FRACTURE

By

Tsvetanka Sendova

and

Jay R. Walton

IMA Preprint Series \# 2327

( July 2010)

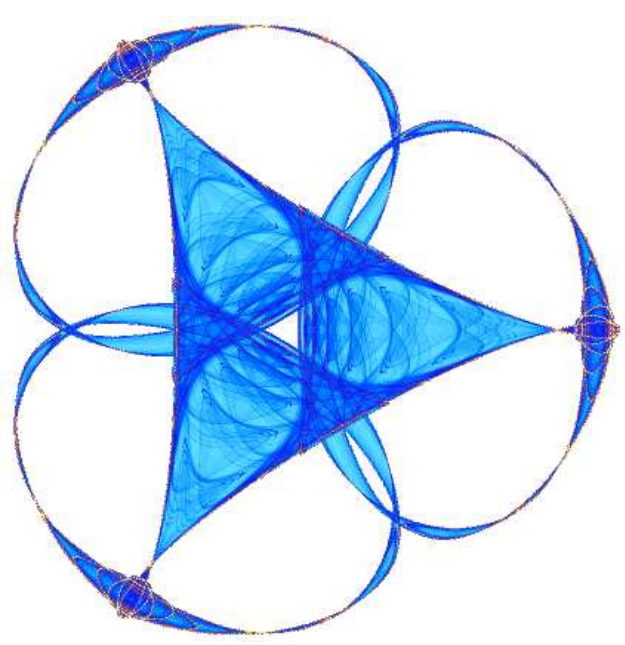

INSTITUTE FOR MATHEMATICS AND ITS APPLICATIONS

UNIVERSITY OF MINNESOTA 400 Lind Hall

207 Church Street S.E.

Minneapolis, Minnesota 55455-0436

Phone: 612-624-6066 Fax: 612-626-7370

URL: http://www.ima.umn.edu 


\title{
The Effect of Surface Tension in Modeling Interfacial Fracture
}

\author{
Tsvetanka Sendova* and Jay R. Walton ${ }^{\dagger}$ \\ *Institute for Mathematics and Its Applications, University of Minnesota, \\ sendovalima.umn. edu \\ †Department of Mathematics, Texas A\&M University, jwal ton@math . tamu. edu
}

\begin{abstract}
In this article the problem of an interface fracture between two isotropic linear elastic materials is studied within a continuum modeling framework, which incorporates important nanoscale effects. The proposed model of bi-material crack ascribes curvature-dependent surface tension to both the fracture surfaces and the solid-solid interface. Further, it uses as boundary conditions the jump momentum balance equations, which take into account the excess properties ascribed to the material interfaces. Ultimately, the model leads to a $4 \times 4$ system of Cauchy-singular integro-differential equations which is equivalent to a system of Fredholm integral equations. It is demonstrated herein, using the Method of Integral Transforms and the Theory of Fredholm Integral equations, that this model leads to bounded stresses and strains, in contrast to the square-root singularities of the stress and strain fields predicted by the classical theory of brittle fracture.
\end{abstract}

Keywords: interfacial fracture, surface tension, Fredholm integral equations PACS: 81

\section{INTRODUCTION}

We consider the problem of interfacial fracture, i.e., fracture occurring in the immediate, nanoscale neighborhood of the interface separating the two phases.

The current work is an extension of the analysis initiated in [1] of a new approach to modeling brittle fracture incorporating nanoscale effects of significance near the fracture surfaces into a continuum theory. This approach avoids the use of any adjustable parameters or ad-hoc assumptions about the crack behavior. In [1] the considered modeling framework was successfully applied to the study of the classical Griffith crack (a crack of finite length in a single linearly elastic material, subjected to far filed tensile loading). It was shown that ascribing curvature-dependent surface tension to the fracture surfaces and using crack surface boundary conditions in the form of the jump momentum balance (which take into account the excess surface properties) leads to a cusp-like crack opening profile at the crack tip, in contrast to the blunt profile predicted by the classical theory of brittle fracture. Moreover, it was demonstrated that such models lead to bounded stresses and strains. In particular, they do not suffer from the internal inconsistencies, arising in Linear Elastic Fracture Mechanics (LEFM), which is based on the assumption of small stresses and strains, but also predicts singular stresses and strains in a neighborhood of a crack tip.

In this paper we consider the problem of an interface fracture between two isotropic linear elastic materials. It is well known that the classical approach of linear elastic fracture mechanics not only predicts stresses and strains with square root singularities 
at the crack tips, but it also yields interpenetration of the crack surfaces ahead of the crack tip. The main goal of this article is to show that the incorporation of curvaturedependent excess properties (e.g. surface tension) into the fracture model removes the stress and strain singularities characteristic of the LEFM approach. This is accomplished by constructing the Dirichlet to Neumann (DtN) and Neumann to Dirichlet (NtD) maps which are then used to derive a $4 \times 4$ system of Cauchy singular integro-differential equations with four compatibility conditions for the derivatives of the displacement vectors evaluated at the fracture surfaces. The system is then recast as a system of Fredholm integral equations, which is shown to have bounded solutions. The details of the analysis are provided in Section 3.

\subsection{Background and Related Work}

Given its importance in fracture mechanics, the plane problem of an interface crack between two dissimilar elastic slabs (or semi-infinite planes) has received significant attention in the literature. The earliest contribution is due to Williams $[2,3]$ where the author demonstrates that the boundary and continuity conditions give rise to a sequence of complex eigenvalues which lead to oscillatory singularities of the displacement and stress fields at the tip of an interface crack (and therefore to interpenetration of the crack surfaces). The problem was later studied by England [4], who deduced a global solution for the classical problem of a uniformly pressurized interface-crack of finite length, as well as by Rice and Sih [5] who derived constraints on the loading parameters, which ensure existence of solutions.

The physically unreasonable behavior of the solutions to the classical interface crack problem prompted attempts to remove the inadmissible field oscillations through new modeling approaches. Comninou $[6,7,8,9]$ sought to remove this unphysical behavior through ad-hoc assumptions that the crack-faces remain in frictionless contact over two sub-segments (adjacent to the two ends of the crack), where the length of these segments was used as an adjustable parameter.

In [10] Knowles and Sternberg aim to remove the oscillatory singularities arising in interface-crack problems by setting the problem within the context of nonlinear theory of elasticity and using a neo-Hookean model to describe the constitutive behavior of the materials. Related work, among many others, also includes results by Chen and Erdogan [11], Suo and Hutchinson [12], Sburiati et al [13], Rice [14], Hutchinson [15], Suo [16], and Shih and Asaro [17].

\section{PROBLEM DESCRIPTION AND NOTATION}

Assume that the body occupies the entire 2-dimensional Euclidean space and consists of two solid phases, denoted by $A$ and $B$, both of which can be modeled as linear elastic solids. Let $\mathscr{B}_{\kappa}$ denote the unloaded configuration of the body, which we choose as the reference configuration. We assume that in the reference configuration phase $A$ occupies the upper half space, while phase $B$ - the lower half space, with a flat interface between the two phases. Further, let there be a crack of finite length at the interface between 


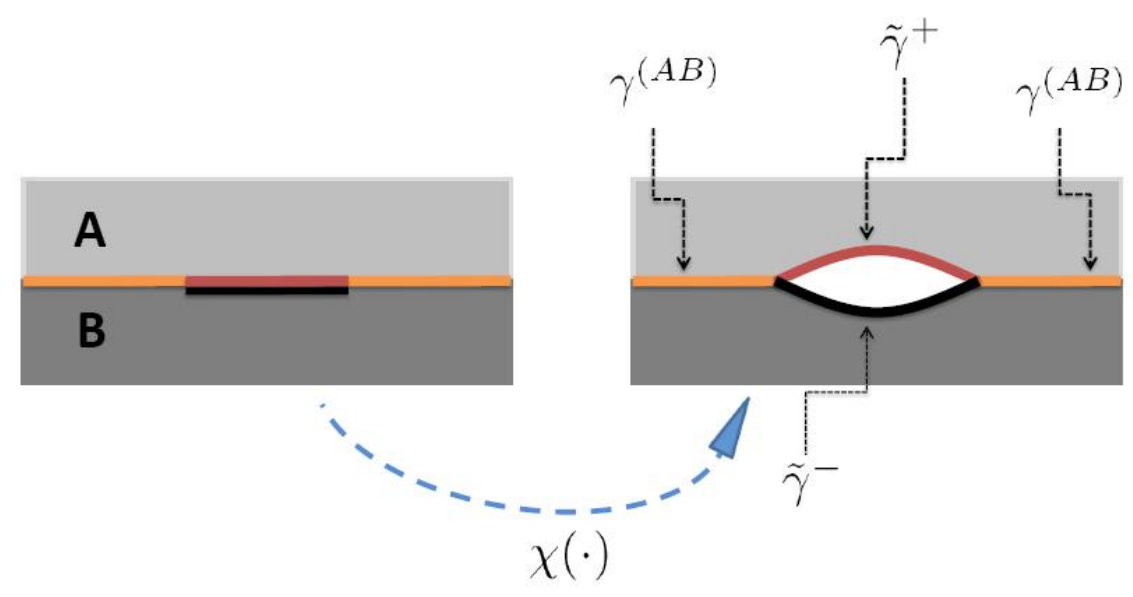

FIGURE 1. An interface crack.

phases $A$ and $B$, which is assumed to close up to a slit in the unloaded configuration. In our case, since the phase interface is assumed to be flat, the crack is a segment (see Fig. 1). The center of this segment is chosen as the origin of a Cartesian coordinate system, and the phase interface - as the $X_{1}$-axis. The positions of points in $\mathscr{B}_{\kappa}$ are labeled by $\mathbf{X}=\left\langle X_{1}, X_{2}\right\rangle$. Without loss of generality, assume that the crack in the reference configuration is parameterized by

$$
\Sigma_{\kappa}^{ \pm}=\left\{\mathbf{X}:-1 \leq X_{1} \leq 1, X_{2}=0^{ \pm}\right\}
$$

Here $\Sigma_{\kappa}^{+}$and $\Sigma_{\kappa}^{-}$denote respectively the upper and lower crack surfaces in the reference configuration. The notational convention used above and in what follows assigns superscript " + " to quantities associated with the upper crack surface, or phase $A$, and superscript "-" to quantities associated with the lower crack surface, or phase $B$.

In addition, let the body be subjected to far-field tensile loading $\sigma$.

A deformation $\chi$ maps points $\mathbf{X}$ in $\mathscr{B}_{\kappa}$ into points $\mathbf{x}$ in the deformed configuration of the body, $\mathscr{B}$, i.e., $\chi: \mathscr{B}_{\kappa} \rightarrow \mathscr{B}$ with $\mathbf{x}=\chi(\mathbf{X})$. The deformed configuration is also referred to as the current or Eulerian configuration of the body, and points $\mathbf{x} \in \mathscr{B}$ are called spatial points, while $\mathbf{X} \in \mathscr{B}_{\kappa}$ are referred to as material points. The material description of the displacement is denoted by $\mathbf{u}(\mathbf{X}):=\chi(\mathbf{X})-\mathbf{X}$, with components $\mathbf{u}(\mathbf{X})=\left\langle u_{1}\left(X_{1}, X_{2}\right), u_{2}\left(X_{1}, X_{2}\right)\right\rangle$. As usual, let $u_{i, j}=\frac{\partial u_{i}}{\partial X_{j}}$.

The gradient and divergence operators are denoted by $\nabla$ and Div respectively in the material frame, and by grad and div in the spatial frame. Similarly, $\nabla_{(\sigma)}$ and $\operatorname{Div}_{(\sigma)}$ denote surface gradient and surface divergence in the material frame, while $\operatorname{grad}_{(\sigma)}$ and $\operatorname{div}_{(\sigma)}$ are the corresponding operators in the spatial frame. ${ }^{1}$

Let $\mathbf{F}(\mathbf{X})$ denote the deformation gradient, i.e. $\mathbf{F}(\mathbf{X})=\nabla \chi(\mathbf{X})$, and let $J=\operatorname{det}(\mathbf{F})$ be its determinant. Also, let $\Sigma^{ \pm}=\chi\left(\Sigma_{\kappa}^{ \pm}\right)$be the upper and lower crack surfaces in the

\footnotetext{
${ }^{1}$ See $[18,1]$ for definitions of surface gradient and surface divergence.
} 
current configuration. It is easy to see that the upper/lower crack surface in the current configuration can be parameterized in terms of the material variables as follows

$$
\Sigma^{ \pm}=\left\{\mathbf{x}: x_{1}=X_{1}+u_{1}\left(X_{1}, 0^{ \pm}\right), x_{2}=u_{2}\left(X_{1}, 0^{ \pm}\right),-1 \leq X_{1} \leq 1\right\} .
$$

Since the body is assumed to be elastic, there exists a symmetric tensor-valued function $\hat{\mathbf{T}}$, such that the stress $\mathbf{T}$ at $\mathbf{x}=\chi(\mathbf{X})$ is given by

$$
\mathbf{T}(\mathbf{x})=\hat{\mathbf{T}}(\mathbf{X}, \mathbf{F}) .
$$

Here $\mathbf{T}$ denotes the Cauchy stress tensor. Let $\mathbf{T}_{\kappa}=J \mathbf{T} \mathbf{F}^{-T}$ be the first Piola-Kirchhoff stress tensor, whose matrix representation in Cartesian coordinates is given by

$$
\left[\mathbf{T}_{\kappa}\right]=\left(\begin{array}{ll}
\sigma_{11} & \sigma_{12} \\
\sigma_{21} & \sigma_{22}
\end{array}\right) .
$$

The material in both phase $A$ and phase $B$ is assumed to be linear elastic. One can show that this is equivalent to modeling the constitutive behavior of the material by Hooke's law in the reference configuration, i.e.,

$$
\mathbf{T}_{\kappa}=2 \mu \mathbf{E}+\lambda \operatorname{tr}(\mathbf{E}) \mathbf{I},
$$

where

$$
\mathbf{E}=\frac{1}{2}\left(\nabla \mathbf{u}+\nabla \mathbf{u}^{T}\right)
$$

is the infinitesimal strain tensor, and

$$
\lambda=\left\{\begin{array}{ll}
\lambda^{+}, & X_{2}>0 \\
\lambda^{-}, & X_{2}<0
\end{array} \quad \mu= \begin{cases}\mu^{+}, & X_{2}>0 \\
\mu^{-}, & X_{2}<0\end{cases}\right.
$$

Here $\lambda^{ \pm}$and $\mu^{ \pm}$are the Lamé moduli of the two solid phases.

While the bulk material behavior is to be modeled within the theory of linear elasticity, one needs to take into account the effects of long range intermolecular forces at the adjoining faces. This is done through the incorporation of excess surface properties (such as surface tension) to the material interfaces. It is assumed that there exists a surface Cauchy stress tensor $\mathbf{T}^{(\sigma)}$ which maps a unit conormal vector to a curve $\mathbf{s}$ in the fracture surface in the current configuration, ${ }^{2}$ to the contact force per unit length exerted across the curve $\mathbf{s}$ upon the material on the negative side of $\mathbf{s}$ by the material on the positive side. Moreover, for simplicity and consistency with the literature, we assume that $\mathbf{T}^{(\sigma)}$ is Eulerian, i.e., $\mathbf{T}^{(\sigma)}=\gamma \mathbf{P}$, where $\mathbf{P}$ is the perpendicular projection onto the tangent space to the fracture surface (in the current configuration). We refer to $\gamma$ as the surface tension (cf. [18]).

\footnotetext{
2 The terminology used here assumes that the body is embedded in 3-dimensional Euclidean space, and that the phase interface is a 2-dimensional surface. The 3-dimensional plane strain problem, however, can be reduced to the 2-dimensional problem considered herein.
} 
The differential and jump momentum balance equations, formulated in the deformed configuration take the following form:

$$
\begin{aligned}
& \operatorname{grad}_{(\sigma)} \gamma^{(A, B)}+2 \gamma^{(A, B)} H \mathbf{n}+[[\mathbf{T}]] \mathbf{n}=\mathbf{0}, \quad \mathbf{x} \in \chi\left(\left\{\mathbf{X}:\left|X_{1}\right|>1, X_{2}=0\right\}\right) \\
& \operatorname{grad}_{(\sigma)} \tilde{\gamma}^{ \pm}+2 \tilde{\gamma}^{ \pm} H \mathbf{n}+[[\mathbf{T}]] \mathbf{n}=\mathbf{0}, \quad \mathbf{x} \in \chi\left(\left\{\mathbf{X}:\left|X_{1}\right|<1, X_{2}=0^{ \pm}\right\}\right)
\end{aligned}
$$

where $[[\mathbf{T}]]$ denotes the jump of the Cauchy stress across the phase interface, in $(6)_{2} \mathbf{n}$ is the unit normal to the fracture surface $\Sigma^{ \pm}$pointing into the bulk material, while in $(6)_{1} \mathbf{n}$ is the unit normal to the solid-solid interface, pointing into phase $A$, and $H=-\frac{1}{2} \operatorname{div}_{(\sigma)} \mathbf{n}$ is the mean curvature. For a detailed derivation of these boundary conditions, see [1].

To find the deformation which the body undergoes under the given loading, one solves a boundary value problem for the components of the displacement vector $\mathbf{u}(\mathbf{X})$, which arises from the balance of linear momentum, the constitutive equations (5), the jump momentum balance conditions (6), as well as the far field loading condition at infinity. Note that $(6)_{2}$ provides the boundary conditions on the crack surfaces, which are highly nonlinear because of the ascribed surface excess properties.

From a mathematical viewpoint, a crack is modeled as a non-self-intersecting curve (in three dimensions - surface) in the reference configuration, across which stresses and strains can suffer a discontinuity. At the same time, it is not entirely clear how to rigorously define a fracture in the deformed configuration. For this reason, and because we will be using the method of integral transforms, which is most easily applied in the unloaded configuration, where the crack is assumed to close to a slit, we formulate the problem in the reference configuration of the body.

Using the pull-back map, one can formulate the jump momentum balance equations (6) in the reference configuration. For simplicity, the notation $\left\langle X_{1}, X_{2}\right\rangle$ will be substituted by $\langle x, y\rangle$ in the equations to follow. Given the complexity of the boundary conditions in their Lagrangian formulation, in what follows we use their linear approximation.

Assume the surface tension $\tilde{\gamma}^{ \pm}(x)$ on the upper and lower crack surfaces has the following asymptotic expansion when stresses and strains remain small:

$$
\tilde{\gamma}^{ \pm}(x)=\gamma_{0}^{ \pm}+\gamma_{1}^{ \pm} u_{2,11}\left(x, 0^{ \pm}\right)+\text {h.o.t. }, \quad|x|<1 .
$$

Thus, the linearized jump momentum balance equations (formulated in the reference configuration) on $|x|<1$, under the assumption that $u_{i, j}\left(x, 0^{ \pm}\right)$and $u_{i, j k}\left(x, 0^{ \pm}\right), i, j, k=$ 1,2 are small, are given by

$$
\left.\begin{array}{l}
\sigma_{12}\left(x, 0^{ \pm}\right)= \pm \gamma_{1}^{ \pm} u_{2,111}\left(x, 0^{ \pm}\right)-\sigma_{12}^{\infty}+\text { h.o.t. } \\
\sigma_{22}\left(x, 0^{ \pm}\right)=\mp \gamma_{0}^{ \pm} u_{2,11}\left(x, 0^{ \pm}\right)-\sigma_{22}^{\infty}+\text { h.o.t. }
\end{array}\right\} \quad|x|<1 .
$$

Here $\sigma_{12}^{\infty}$ and $\sigma_{22}^{\infty}$ denote the shear and tensile far-field loading parameters respectively.

The solution satisfies the following bond conditions, which ensure continuity of displacements along the bonded portion of the interface

$$
u_{i}\left(x, 0^{+}\right)=u_{i}\left(x, 0^{-}\right), \quad i=1,2 \quad|x|>1
$$


and linearized jump momentum balance equations on $|x|>1$

$$
\left.\begin{array}{l}
\sigma_{12}\left(x, 0^{+}\right)-\sigma_{12}\left(x, 0^{-}\right)=\gamma_{1}^{(A, B)} u_{2,111}(x, 0)+\text { h.o.t. }, \\
\sigma_{22}\left(x, 0^{+}\right)-\sigma_{22}\left(x, 0^{-}\right)=-\gamma_{0}^{(A, B)} u_{2,11}(x, 0)+\text { h.o.t. }
\end{array}\right\} \quad|x|>1,
$$

where $\gamma^{(A, B)}(x)=\gamma_{0}^{(A, B)}+\gamma_{1}^{(A, B)} u_{2,11}(x, 0)+$ h.o.t., for $|x|>1$, is the surface tension ascribed to the interface between phases $A$ and $B$ (see Fig. 1).

\section{METHOD OF INTEGRAL TRANSFORMS}

As in the case of the classical Griffith crack (cf. [1]), using integral transform techniques one constructs the Dirichlet to Neumann map for the problem

$$
\begin{aligned}
& \sigma_{12}\left(x, 0^{ \pm}\right)=A^{ \pm} u_{2,1}\left(x, 0^{ \pm}\right) \pm B^{ \pm} \mathscr{H}\left[u_{1,1}\right]\left(x, 0^{ \pm}\right), \quad-\infty<x<\infty \\
& \sigma_{22}\left(x, 0^{ \pm}\right)=-A^{ \pm} u_{1,1}\left(x, 0^{ \pm}\right) \pm B^{ \pm} \mathscr{H}\left[u_{2,1}\right]\left(x, 0^{ \pm}\right), \quad-\infty<x<\infty .
\end{aligned}
$$

In a similar way, the Neumann to Dirichlet map is given by

$$
\begin{aligned}
& u_{1,1}\left(x, 0^{ \pm}\right)=C^{ \pm} \sigma_{22}\left(x, 0^{ \pm}\right) \mp D^{ \pm} \mathscr{H}\left[\sigma_{12}\right]\left(x, 0^{ \pm}\right), \quad-\infty<x<\infty \\
& u_{2,1}\left(x, 0^{ \pm}\right)=-C^{ \pm} \sigma_{12}\left(x, 0^{ \pm}\right) \mp D^{ \pm} \mathscr{H}\left[\sigma_{22}\right]\left(x, 0^{ \pm}\right), \quad-\infty<x<\infty,
\end{aligned}
$$

where $A^{ \pm}, B^{ \pm}, C^{ \pm}$, and $D^{ \pm}$are given explicitly in terms of the Lamé constants of the two phases:

$$
A^{ \pm}=\frac{2\left(\mu^{ \pm}\right)^{2}}{\lambda^{ \pm}+3 \mu^{ \pm}}, B^{ \pm}=\frac{2 \mu^{ \pm}\left(\lambda^{ \pm}+2 \mu^{ \pm}\right)}{\lambda^{ \pm}+3 \mu^{ \pm}}, C^{ \pm}=\frac{1}{2\left(\lambda^{ \pm}+\mu^{ \pm}\right)}, D^{ \pm}=\frac{\lambda^{ \pm}+2 \mu^{ \pm}}{2 \mu^{ \pm}\left(\lambda^{ \pm}+\mu^{ \pm}\right)},
$$

and $\mathscr{H}[f](x)$ is the Hilbert transform of $f(x)$, namely

$$
\mathscr{H}[f](x)=\frac{1}{\pi} f_{-\infty}^{\infty} \frac{f(r)}{r-x} d r
$$

Here $f$ denotes a Cauchy principal value integral.

In the classical model there are no excess properties ascribed to the interfaces, and therefore, we have equality of stresses along the whole $x$-axis. This allows one to formulate the problem in terms of the displacement jumps and derive a $2 \times 2$ system of Cauchy singular integro-differential equations for the two displacement jumps, which has been well studied $[2,3,4,5]$. Due to the specific boundary conditions, however, the considered model leads to a $4 \times 4$ system of singular integro-differential equations for the four unknown functions $u_{1,1}\left(x, 0^{+}\right), u_{1,1}\left(x, 0^{-}\right), u_{2,1}\left(x, 0^{+}\right)$and $u_{2,1}\left(x, 0^{-}\right)$.

As compared to the problem of a fracture in a single material, the governing equations turn out to be much more complicated - all four unknown functions are coupled, and the equations involve third order derivatives of the slope of the crack profile of the upper and lower crack surfaces $u_{2,1}\left(x, 0^{ \pm}\right)$, as well as second order derivatives of $u_{1,1}\left(x, 0^{ \pm}\right)$. 
To simplify the notation, it is convenient to introduce

$$
\begin{array}{ll}
\phi_{1}^{ \pm}(x)=u_{1,1}\left(x, 0^{ \pm}\right) & \psi_{1}^{ \pm}(x)=u_{1,111}\left(x, 0^{ \pm}\right)=\left(\phi_{1}^{ \pm}\right)^{\prime \prime}(x) \\
\phi_{2}^{ \pm}(x)=u_{2,1}\left(x, 0^{ \pm}\right) & \psi_{2}^{ \pm}(x)=u_{2,1111}\left(x, 0^{ \pm}\right)=\left(\phi_{2}^{ \pm}\right)^{\prime \prime \prime}(x) .
\end{array}
$$

Let us assume $\sigma_{12}^{\infty}=0$. In this case $\phi_{1}^{ \pm}(x)$ are even functions, whereas $\phi_{2}^{ \pm}(x)$ are odd functions on $[-1,1]$. We seek solutions of the governing system of equations satisfying

$$
\phi_{1}^{ \pm}(-1)=\phi_{2}^{ \pm}(-1)=\left(\phi_{2}^{ \pm}\right)^{\prime}(-1)=0 .
$$

One can show that the above mentioned $4 \times 4$ system of Cauchy singular integrodifferential equations is equivalent to the following $4 \times 4$ system of Fredholm integral equations (14)-(17) in terms of the highest order derivatives $\psi_{1}^{ \pm}(x)=u_{1,111}\left(x, 0^{ \pm}\right)$and $\psi_{2}^{ \pm}(x)=u_{2,1111}\left(x, 0^{ \pm}\right)$, together with four compatibility conditions (18)-(21). This is achieved by first reducing the system to canonical form, i.e. into a system of singular equations for the terms with highest order derivatives and subsequently showing that the singular integral operators involved in the considered equations can be recast as Fredholm integral operators.

$$
\begin{gathered}
\frac{\gamma_{1}^{+}}{B^{+}} \psi_{2}^{+}(x)-\frac{\gamma_{1}^{-}}{B^{-}} \psi_{2}^{-}(x)=\frac{c_{1}^{+}-c_{1}^{-}}{\pi} \ln \left(1-x^{2}\right)-\frac{1}{\pi} \int_{-1}^{1}\left(\psi_{1}^{+}(r)-\psi_{1}^{-}(r)\right) \ln |r-x| d r \\
+\frac{A^{+}}{B^{+}}\left[\int_{-1}^{x}(x-r) \psi_{2}^{+}(r) d r-c_{2}^{+}(1+x)\right]+\frac{A^{-}}{B^{-}}\left[\int_{-1}^{x}(x-r) \psi_{2}^{-}(r) d r-c_{2}^{-}(1+x)\right] \\
\quad-\frac{\gamma_{0}^{+}}{B^{+}} \psi_{2}^{+}(x)+\frac{\gamma_{0}^{-}}{B^{-}} \psi_{2}^{-}(x)+\frac{A^{+}}{B^{+}} \psi_{1}^{+}(x)+\frac{A^{-}}{B^{-}} \psi_{1}^{-}(x) \\
=\frac{1}{\pi}\left(c_{2}^{+}-c_{2}^{-}\right) \ln \left(1-x^{2}\right)-\frac{1}{\pi} \int_{-1}^{1}\left(\psi_{2}^{+}(r)-\psi_{2}^{-}(r)\right) \ln |r-x| d r \\
\left(\frac{C^{+}}{D^{+}} \gamma_{1}^{+}+\frac{\gamma_{0}^{+} \gamma_{0}^{(A, B)}}{2 B^{+}}\right) \psi_{2}^{+}(x)-\left(\frac{C^{-}}{D^{-}} \gamma_{1}^{-}-\frac{\gamma_{0}^{-} \gamma_{0}^{(A, B)}}{2 B^{-}}\right) \psi_{2}^{-}(x) \\
-\frac{\gamma_{0}^{(A, B)}}{2}\left(\frac{A^{+}}{B^{+}} \psi_{1}^{+}(x)-\frac{A^{-}}{B^{-}} \psi_{1}^{-}(x)\right) \\
=-\frac{1}{D^{+}}\left[\int_{-1}^{x}(x-r) \psi_{2}^{+}(r) d r-c_{2}^{+}\right]-\frac{1}{D^{-}}\left[\int_{-1}^{x}(x-r) \psi_{2}^{-}(r) d r-c_{2}^{-}\right] \\
+\frac{1}{\pi}\left(\left(\gamma_{0}^{+}-\frac{\gamma_{0}^{(A, B)}}{2}\right) c_{2}^{+}+\left(\gamma_{0}^{-}-\frac{\gamma_{0}^{(A, B)}}{2}\right) c_{2}^{-}\right) \ln \left(1-x^{2}\right) \\
-\frac{1}{\pi} \int_{-1}^{1}\left(\left(\gamma_{0}^{+}-\frac{\gamma_{0}^{(A, B)}}{2}\right) \psi_{2}^{+}(r)+\left(\gamma_{0}^{-}-\frac{\gamma_{0}^{(A, B)}}{2}\right) \psi_{2}^{-}(r)\right) \ln |r-x| d r
\end{gathered}
$$




$$
\begin{aligned}
& \frac{\gamma_{1}^{(A, B)}}{2}\left(\frac{A^{+}}{B^{+}} \psi_{1}^{+}(x)-\frac{A^{-}}{B^{-}} \psi_{1}^{-}(x)-\frac{\gamma_{0}^{+}}{B^{+}} \psi_{2}^{+}(x)-\frac{\gamma_{0}^{-}}{B^{-}} \psi_{2}^{-}(x)\right) \\
& =+\frac{1}{\pi} \int_{-1}^{1}\left(\left(\gamma_{1}^{+}-\frac{\gamma_{1}^{(A, B)}}{2}\right) \psi_{2}^{+}(r)+\left(\gamma_{1}^{-}-\frac{\gamma_{1}^{(A, B)}}{2}\right) \psi_{2}^{-}(r)\right) \ln |r-x| d r \\
& -\frac{C^{+}}{D^{+}} \gamma_{0}^{+}\left[\int_{-1}^{x}(x-r) \psi_{2}^{+}(r) d r-c_{2}^{+}(1+x)\right]+\frac{C^{-}}{D^{-}} \gamma_{0}^{-}\left[\int_{-1}^{x}(x-r) \psi_{2}^{-}(r) d r-c_{2}^{-}(1+x)\right] \\
& -\frac{1}{D^{+}}\left[\int_{-1}^{x}(x-r) \psi_{1}^{+}(r) d r-c_{1}^{+}(1+x)\right]-\frac{1}{D^{-}}\left[\int_{-1}^{x}(x-r) \psi_{1}^{-}(r) d r-c_{1}^{-}(1+x)\right] \\
& -\frac{1}{\pi}\left(\left(\gamma_{1}^{+}-\frac{\gamma_{1}^{(A, B)}}{2}\right) c_{2}^{+}+\left(\gamma_{1}^{-}-\frac{\gamma_{1}^{(A, B)}}{2}\right) c_{2}^{-}\right) \ln \left(1-x^{2}\right)+\left(\frac{C^{+}}{D^{+}}+\frac{C^{-}}{D^{-}}\right) \sigma_{22}^{\infty} .
\end{aligned}
$$

Here $c_{1}^{ \pm}=u_{1,11}\left(1,0^{ \pm}\right)$and $c_{2}^{ \pm}=u_{2,111}\left(1,0^{ \pm}\right)$. These boundary values are unknowns, which must be determined from the following four compatibility conditions.

$$
\begin{aligned}
& \int_{0}^{1}\left(\frac{\gamma_{1}^{+}}{B^{+}} \psi_{2}^{+}(x)-\frac{\gamma_{1}^{-}}{B^{-}} \psi_{2}^{-}(x)\right) \sqrt{1-x^{2}} d x+\frac{\pi}{4}\left(\frac{A^{+}}{B^{+}} c_{2}^{+}+\frac{A^{-}}{B^{-}} c_{2}^{-}\right)-c_{1}^{+}+c_{1}^{-} \\
&= \int_{0}^{1} \int_{0}^{x}\left(\frac{A^{+}}{B^{+}} \psi_{2}^{+}(x)+\frac{A^{-}}{B^{-}} \psi_{2}^{-}(x)\right)(x-r) \sqrt{1-r^{2}} d r d x \\
&+ \frac{1}{3} \int_{0}^{1}\left(\frac{A^{+}}{B^{+}} \psi_{2}^{+}(x)+\frac{A^{-}}{B^{-}} \psi_{2}^{-}(x)\right) d x-\frac{1}{2} \int_{0}^{1}\left(1+x^{2}\right)\left(\psi_{2}^{+}(x)-\psi_{2}^{-}(x)\right) d x \\
&-\frac{\pi}{2}\left(\frac{1}{B^{+}}+\frac{1}{B^{-}}\right) \sigma_{22}^{\infty}+\frac{\pi}{2}\left(\frac{\gamma_{0}^{+}}{B^{+}} c_{2}^{+}-\frac{\gamma_{0}^{-}}{B^{-}} c_{2}^{-}-\frac{A^{+}}{B^{+}} c_{1}^{+}-\frac{A^{-}}{B^{-}} c_{1}^{-}\right) \\
&=\int_{0}^{1}\left(\frac{\gamma_{0}^{+}}{B^{+}} \psi_{2}^{+}(x)-\frac{\gamma_{0}^{-}}{B^{-}} \psi_{2}^{-}(x)-\frac{A^{+}}{B^{+}} \psi_{1}^{+}(x)-\frac{A^{-}}{B^{-}} \psi_{1}^{-}(x)\right)\left(\sqrt{1-x^{2}}+x \arcsin (x)\right) d x \\
&-\frac{1}{3} \int_{0}^{1}\left(\frac{1}{D^{+}} \psi_{2}^{+}(x)+\frac{1}{D^{-}} \psi_{2}^{-}(x)\right) d x+\frac{\pi}{4}\left(\frac{1}{D^{+}} c_{2}^{+}+\frac{1}{D^{-}} c_{2}^{-}\right) \\
&=\int_{0}^{1}\left(\frac{1}{D^{+}} \psi_{2}^{+}(x)+\frac{1}{D^{-}} \psi_{2}^{-}(x)\right)\left(\int_{0}^{x}(x-r) \sqrt{1-r^{2}} d r\right) d x \\
&+\int_{0}^{1}\left(\left(\frac{C^{+}}{D^{+}} \gamma_{1}^{+}+\frac{\gamma_{0}^{(A, B)} \gamma_{0}^{+}}{2 B^{+}}\right) \psi_{2}^{+}(x)+\left(-\frac{C^{-}}{D^{-}} \gamma_{1}^{-}+\frac{\gamma_{0}^{(A, B)} \gamma_{0}^{-}}{2 B^{-}}\right) \psi_{2}^{-}(x)\right. \\
&\left.-\frac{\gamma_{0}^{(A, B)}}{2}\left(\frac{A^{+}}{B^{+}} \psi_{1}^{+}(x)-\frac{A^{-}}{B^{-}} \psi_{1}^{-}(x)\right)\right) \sqrt{1-x^{2}} d x
\end{aligned}
$$




$$
\begin{aligned}
& \int_{0}^{1}\left(\frac{1}{D^{+}} \psi_{1}^{+}(x)+\frac{1}{D^{-}} \psi_{1}^{-}(x)+\frac{C^{+}}{D^{+}} \gamma_{0}^{+} \psi_{2}^{+}(x)-\frac{C^{-}}{D^{-}} \gamma_{0}^{-} \psi_{2}^{-}(x)\right)\left(\sqrt{1-x^{2}}+x \arcsin (x)\right) d x \\
& +\frac{\gamma_{1}^{(A, B)}}{2} \int_{0}^{1}\left(\frac{A^{+}}{B^{+}} \psi_{1}^{+}(x)-\frac{A^{-}}{B^{-}} \psi_{1}^{-}(x)-\frac{\gamma_{0}^{+}}{B^{+}} \psi_{2}^{+}(x)-\frac{\gamma_{0}^{-}}{B^{-}} \psi_{2}^{-}(x)\right) \frac{1}{\sqrt{1-x^{2}}} d x \\
& =-\frac{\pi}{2}\left(\frac{C^{+}}{D^{+}}+\frac{C^{-}}{D^{-}}\right) \sigma_{22}^{\infty}+\frac{\pi}{2}\left(\frac{1}{D^{+}} c_{1}^{+}+\frac{1}{D^{-}} c_{1}^{-}+\frac{C^{+}}{D^{+}} c_{2}^{+}-\frac{C^{-}}{D^{-}} c_{2}^{-}\right)
\end{aligned}
$$

As a system of Fredholm integral equations, (14)-(17) has solutions $\psi_{1}^{ \pm}(x), \psi_{2}^{ \pm}(x)$ that have at most logarithmic singularities at $x= \pm 1$. Their first integrals are then continuous on $[-1,1]$, from where it follows that stress and strain are bounded at the crack tips $x= \pm 1$. Note that equations (14)-(17) and the four compatibility conditions (18)-(21) for $c_{1}^{ \pm}$and $c_{2}^{ \pm}$must be solved simultaneously.

\section{CONCLUDING REMARKS}

The problem of interfacial fracture and the related problem of debonding of a composite material body are important ones in engineering applications. The classical approach to modeling interface cracks, based on LEFM leads not only to square root singularities of the stress and strain fields in a neighborhood of the crack tip, but also the boundary and continuity conditions give rise to a sequence of complex eigenvalues which lead to oscillatory singularities of the displacement and stress fields at the tip of an interface crack (and therefore to interpenetration of the crack surfaces) (cf. [2, 3]).

This paper studies the effect of correcting the bulk material constitutive behavior due to the proximity of neighboring phase of a different material, through ascribing curvature-dependent surface tension to the fracture surfaces and the solid-solid interface. As shown above, this new modeling approach yields bounded stresses and strains, thus resolving the singular stress behavior predicted by LEFM.

It remains to investigate whether the incorporation of excess properties of the dividing surfaces also removes the unphysical effects of interpenetration.

\section{ACKNOWLEDGMENTS}

Tsvetanka Sendova would like to gratefully acknowledge the support of the Institute for Mathematics and Its Applications.

\section{REFERENCES}

1. T. Sendova, and J. Walton, Mathematics and Mechanics of Solids 15, 368 (2010).

2. M. L. Williams, ASME Journal of Applied Mechanics 24, 109-114 (1957).

3. M. L. Williams, Bulletin of the Seismological Society of America 49, 199-204 (1959).

4. A. H. England, Journal of Applied Mechanics 32, 400-402 (1965). 
5. J. R. Rice, and G. C. Sih, ASME J. Appl. Mech 32, 418-423 (1965).

6. M. Comninou, appl. Mech 44, 631-636 (1977).

7. M. Comninou, ASME, Transactions, Journal of Applied Mechanics 45, 287-290 (1978).

8. M. Comninou, and J. Dundurs, Journal of Elasticity 10, 203-212 (1980).

9. M. Comninou, Engineering Fracture Mechanics 37, 197-208 (1990).

10. J. K. Knowles, and E. Sternberg, Journal of Elasticity 13, 257-293 (1983).

11. Y. F. Chen, and F. Erdogan, Journal of the Mechanics and Physics of Solids 44, 771-787 (1996).

12. Z. Suo, and J. Hutchinson, International Journal of Fracture 43, 1-18 (1990).

13. R. Sburiati, E. Madenci, and I. Guven, Journal of Applied Mechanics 67, 590 (2000).

14. J. Rice, J. Appl. Mech.(Trans. ASME) 55, 98-103 (1988).

15. J. Hutchinson, Metal-Ceramic Interfaces pp. 295-301 (1990).

16. Z. Suo, Mechanics of interface fracture, Ph.D. thesis, Harvard University (1989).

17. C. F. Shih, and R. J. Asaro, International Journal of Fracture 42, 101-116 (1990).

18. J. C. Slattery, L. Sagis, and E. S. Oh, Interfacial Transport Phenomena, Springer-Verlag, 2007, 2 edn. 